\title{
ANTIMICROBIAL RESISTANCE AND MOLECULAR TYPING OF Salmonella spp. ISOLATED FROM POULTRY IN
} KHARTOUM STATE, SUDAN

\author{
Hassan Elsiddig Hag Elsafi*1, Mohamed Amin Mohamed Siddig ${ }^{2}$, Anne ${ }^{3}$ Muckle, Jaime Martinez-Urtaza ${ }^{4}$ \\ Address (es): \\ ${ }^{1}$ Ahfad Centre for Science and Technology, Ahfad University for Women, Omdurman, Sudan. \\ ${ }^{2}$ Department of Botany, Faculty of Science, University of Khartoum, Khartoum, Sudan. \\ ${ }^{3}$ Pathology and Microbiology, Atlantic Veterinary College, University of Prince Edward Island, Charlottetown, Prince Edward Island, Canada \\ ${ }^{4}$ Department of Genetics and Microbiology, Facultat de Biociències - Edifici C, Universitat Autònoma de Barcelona (UAB), Barcelona $\cdot$ Spain
}

*Corresponding author: hassanelsafi@gmail.com

\section{ABSTRACT}

https://doi.org/10.36547/be.363

\section{ABSTRACT}

Salmonella is considered one of the main foodborne pathogens. In this study a total of 38 Salmonella isolates were recovered from 679 (5.6\%) specimens collected within Khartoum State. The specimens included, intestinal contents $11.4 \%(14 / 160)$ egg 7\% (7/99), liver 5\% (5/100) skin 5\% (10/200), feed 0\% (0/40\%), water 0\% (0/100). Serotyping revealed the presence of eight serovars: Kentucky 11 (28.9\%), Stanleyville 8 (21.05\%), Virchow 6 (16\%), Alachua 5 (13.16\%), Blockley 4 (10.53\%), Hadar $2(5.26 \%)$, Typhimurium $1(2.63 \%)$ and Havana $1(2.63 \%)$. Antibiotic resistance profile, using disc diffusion method, indicated that all isolates were sensitive to apramycin, chloramphenicol, cefoperazone and cefotaxime. The 38 isolates were found to be resistant to tetracycline (52.6\%), nalidixic acid (50.0\%), compound sulfonamide (44.7\%), sulfamethoxazole-trimethoprim (31.6\%), streptomycin (26.3\%), gentamycin (15.8\%), neomycin (15.8\%), furazolidone (7.9\%), ampicillin (5.3\%), ciprofloxacin (5.3\%), amikacin (2.6\%), amoxicillin-clavulanic acid (2.6\%), ceftazidime (2.6\%), and colistin (2.6\%) in decreasing order. Only 20 isolates (52.6\%) demonstrated multiple drug resistance. Four of the Salmonella isolates were untypable by XbaI Pulsed Field Gel Electrophoresis (PFGE) technique however the 33 typed isolates were differentiated into 22 PFGE patterns. This study aimed to determine the prevalence, antimicrobial resistance and molecular typing of Salmonella spp. form poultry

Keywords: Salmonella, Antimicrobial resistance, Poultry, PFGE, PCR, Khartoum State, Sudan

\section{INTRODUCTION}

Salmonella sp. is one of the main sources of foodborne illnesses worldwide (Fung et al., 2018). The main sources of Salmonella infections are associated with consumption of meats, milk and eggs (Jajere, 2019). Salmonella comprises more than 2600 serovars (Shi et al., 2015). Generally there are two groups of Salmonella, typhoidal and non-typhoidal Salmonella. While typhoidal Salmonella can result in systemic infections with high fatal capabilities, non-typhoidal Salmonella infections are generally self-limiting (Su and Chiu, 2007; Gal-Mor $e t$ al., 2014).

The widespread use of antibiotics poses problems for antimicrobial resistance, which leads to an increase in treatment costs and even to therapy failure (Hur $\boldsymbol{e t}$ al., 2011). Multiple drug resistance (MDR) among Salmonella is prevalent. The selective pressure caused by the application of antimicrobials in poultry production and veterinary practice for growth promotion and prophylaxis has resulted in an increase in antibiotic resistance and an increase in the presence of genes conferring antimicrobial resistance to Salmonella (Zishiri et al., 2016). Strains that have been detected are resistant to some clinical first line antibiotics used in the treatment of severe Salmonella infections (Tadee et al., 2015). As antibiotic resistant bacteria can be directly transmitted through the food chain or transfer their antimicrobial resistance to human pathogens by mobile genetic elements, it is important to monitor antibiotic resistance among Salmonella isolates and control the risk.

The purpose of this study was to determine the prevalence, antimicrobial resistance and molecular typing of Salmonella isolates obtained from poultry in Khartoum State, Sudan.

\section{MATERIALS AND METHODS}

\section{Sample collection and Salmonella isolation}

A total of 679 samples of livers, intestinal contents, skin swabs and egg specimens were collected in Khartoum State between October 2004 to May 2005. Samples were selectively enriched in Selenite Cystine broth (Merck KGaA, Darmstadt, Germany) incubated at $37^{\circ} \mathrm{C}$ for $24 \mathrm{~h}$. then were streaked onto Xylose Lysine Desoxycholate agar (Oxoid, Basingstoke, Hampshire, UK) and incubated at $37^{\circ} \mathrm{C}$ for $24 \mathrm{~h}$. Suspected Salmonella isolates were identified using API20E test strips (BioMerieux, France) and confirmed by polymerase chain reaction (PCR) amplification using invA as the target gene (Malorny et al., 2003).

\section{Serotyping}

The Salmonella isolates obtained in this study were submitted to the Office International des Épizotties (OIÉ) Reference laboratory for Salmonellosis, Public Health Agency of Canada (PHAC), Guelph, Ontario, Canada for serotyping. Serological characterization was performed at the $\mathrm{O}$ (somatic) and $\mathrm{H}$ (Flagellar) antigens levels. The tests for serological identification were carried out by the slide agglutination using a micro-technique that employs microtitre plates. Naming of the identified serovars was made according to the antigenic formulae proposed by Popoff (2001).

\section{Antimicrobial susceptipility testing}

Salmonella isolates were tested by the disc diffusion method for antimicrobial susceptibility following the guidelines of the National Committee for Clinical Laboratory Standards National Committee for Clinical Laboratory Standards, (NCCLS, 2000). The antimicrobial drugs tested were amikacin, amoxicillinclavulanic acid, ampicillin, apramycin, chloramphenicol, cefoperazone, ceftazidime, cefotaxime, colistin, furazolidone, gentamicin, nalidixic acid, ciprofloxacin, neomycin, streptomycin, sulfamethoxazole-trimethoprim, compound sulfonamide and tetracycline.

\section{Pulsed filed gel electrophoresis (PFGE)}

PFGE was performed according to the protocol of the Centers for Disease Control and Prevention (CDC) PulseNet protocol (CDC, 2002). Agarose-embedded DNA was digested with $50 \mathrm{U}$ of the enzyme XbaI (Promega, Southampton, UK). DNA restriction fragments were separated by PFGE on $1 \%$ SeaKem Gold agarose (Cambrex, Bio Science Rockland Inc., USA) using 0.5X Tris-Borate-EDTA extended-range buffer (Bio-Rad, Hercules, USA) with recirculation at $14 \mathrm{WC}$ in a CHEF DRIII system (Bio-Rad, Hercules, USA). DNA from Salmonella Braenderup $\mathrm{H} 9812$ restricted with $\mathrm{XbaI}$ was used as a size marker. Pulse times were ramped from 2.2 to $63.8 \mathrm{~s}$ over $18 \mathrm{~h}$ with an angle of $120 \mathrm{~W}$ at $6.0 \mathrm{~V} \mathrm{~cm} \_1$. Genomic-DNA profiles or 'fingerprints' were analyzed using BioNumerics software (Applied Maths, Sint-Martens-Latem, Belgium). 


\section{RESULTS}

\section{Presence of Salmonella spp.}

During this study $5.6 \%(38 / 679)$ Salmonella isolates were isolated. Intestinal contents $11.4 \%$ (14/160) egg 7\% (7/99), liver 5\% (5/100) skin 5\% (10/200), feed $0 \%(0 / 40 \%)$, water $0 \%(0 / 100)$.

\section{Serotyping}

The results obtained led to recognition of eight serovars within the 38 isolates. The detected serovars were: Kentucky, 11 (29.0\%), Stanleyville, 8 (21.1\%), Virchow, 6 (16.0\%), Alachua, 5 (13.2\%), Blockley, 4 (10.5\%), Hadar, 2 (5.3\%), Typhimurium, 1 (2.6\%) and Havana, 1 (2.6\%).

\section{Antimicrobial susceptipility of Salmonella isolates}

The 38 isolates were found to be resistant to tetracycline $(52.6 \%)$, nalidixic acid (50.0\%), compound sulfonamide (44.7\%), sulfamethoxazole-trimethoprim (31.6\%), streptomycin (26.3\%), gentamycin (15.8\%), neomycin (15.8\%), furazolidone (7.9\%), ampicillin (5.3\%), ciprofloxacin (5.3\%), amikacin (2.6\%), amoxicillin-clavulanic acid $(2.6 \%)$, ceftazidime $(2.6 \%)$, and colistin $(2.6 \%)$ in decreasing order (Fig 1).

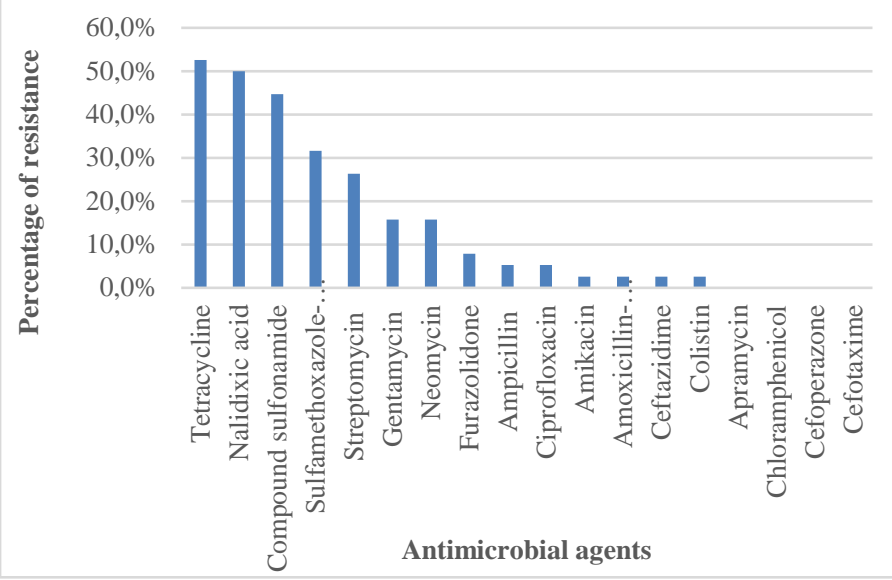

Figure 1 A histogram showing percent resistance of Salmonella isolates to 18 different antimicrobial agents.

Table 1 Multiple drug resistance pattern of the Salmonella isolates.

\begin{tabular}{ccc}
\hline Isolate No. & Serovar & Pattern of resistance \\
\hline S.2 & Kentucky & TE,CN,NA,S \\
S.5 & Virchow & TE,NA,FR,SXT,CS \\
S.6 & Virchow & TE,NA,FR,SXT,CS \\
S.7 & Virchow & TE,NA,FR,SXT,CS \\
S.8 & Alachua & CAZ, CT, AMP \\
S.9 & Blockley & TE, N, NA, ST \\
S.10 & Stanleyville & TE, SXT, CS \\
S.12 & Stanleyville & N, SXT, CS \\
S.13 & Kentucky & TE, CN, NA, ST, CS \\
S.14 & Kentucky & TE, CN, NA, ST, CS \\
S.16 & Virchow & TE, NA, SXT, CS \\
S.17 & Virchow & TE, NA, SXT, CS \\
S.22 & Stanleyville & N, ST, AMP \\
S.23 & Stanleyville & TE, NA, SXT, CS \\
S.25 & Stanleyville & TE, SXT, CS \\
S.27 & Kentucky & TE, CN, NA, ST, SXT, CS \\
S.31 & Kentucky & TE, CN, NA, ST, CS \\
S.34 & Kentucky & TE, CN, NA, ST, CS \\
S.37 & Virchow & TE, NA, SXT, CS \\
S.38 & Havana & TE, SXT, CS \\
\hline TET Tertid
\end{tabular}

TE,Tetracycline; N, Neomycin; CN, Gentamicin; NA, Nalidixic acid; FR, Furazolidone; ST, Streptomycin; SXT, Sulfamethoxazole-trimethoprim;; CAZ, Ceftazidime; CT, Colistin; AMP, Ampicillin.

None of the isolates was found to be resistant to all of the antimicrobial agents used in this study while five isolates; were susceptible to all the 18 different antimicrobial agents used. All of the Salmonella isolates were susceptible to chloramphenicol, cefoperazone, cefotaxime and apramycin.

Table 1 shows the multiple drug resistance profile for some of the Salmonella isolates. Multiple drug resistance was recorded whenever an isolate was found to be resistant to three or more antibiotics. Among the 38 isolates tested for antibiotic susceptibility, 20 isolates (52.6\%) demonstrated multiple drug resistance. Multidrug resistance was predominantly seen among the most prevalent serovars mainly Virchow (15.8\%), Kentucky (15.8\%) and Stanleyville (10.5\%). Only one isolate $(2.6 \%)$ of each of the serovars Blockley, Alachua and Havana showed multidrug resistance.

\section{PFGE analysis}

The 38 Salmonella isolates collected in this study were subjected to PFGE analysis. The fingerprints generated by PFGE using restriction enzyme XbaI. Four isolates did not produce any restriction bands even with repeated trials with other isolates and the standard control. In isolates S.1, S.6 and S.11 apparently, there is a problem with plug preparation while in S.7 the plug was nicely prepared, however, no restriction bands were observed

Fig.2 is a dendrogram produced by BioNumerics soft ware. Analysis of $\mathrm{Xba \textrm {I }}$ restricted DNA of all the Salmonella isolates in this study. Twenty two PFGE patterns (X1 to X22) were observed among the 34 typable Strains. XbaI restriction bands of the isolates were typically composed of 11 to 16 fragments; the most common patterns are $\mathrm{X} 1$ and $\mathrm{X} 4$ each comprised of 3 strains. Considerable genetic diversity clearly exists among the strains. Fig 2 shows that the percentage of similarity between different patterns ranged between 58.9 to $100 \%$. Nine profiles with $100 \%$ similarity are composed of isolates of the same serotype. Moreover, PFGE showed consistency of PFGE profiles with serotyping as no isolates within the same PFGE pattern were of different serotypes.

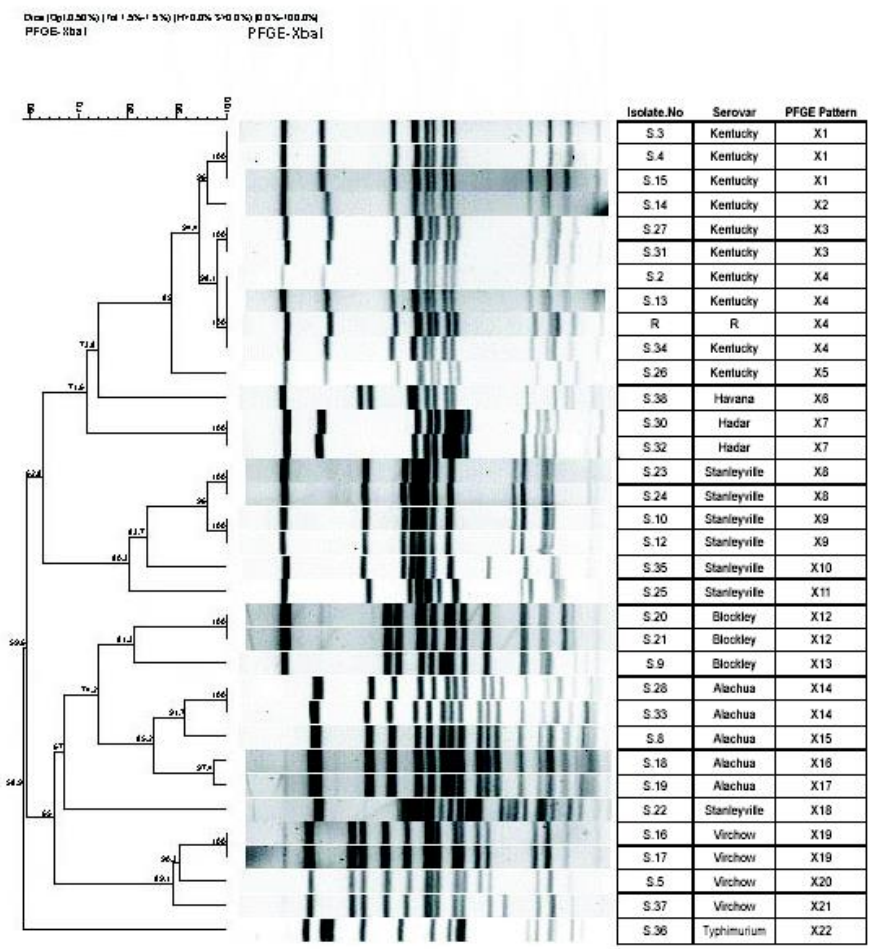

Figure 2 Represantative XbaI PFGE patterns and clustering analysis of Salmonella isolates recovered from poultry. Isolate. No, Serovars identified and PFGE pattern are indicated at the right. Dendrogram was generated by BioNumerics software using Dice coefficient and unweighted pair group method with arithmetic averages (UPGMA). Numbers at dendrogram roots show similarity value (similarity percentage) among identified clusters 


\section{DISCUSSION}

Prevalence of Salmonella in poultry, $5.6 \%$ in this study, is comparably higher than most earlier reported figures for prevalence in Sudan. While Yagoub (1986) reported a $3.9 \%$ prevalence, Fadlalla (2003) reported prevalence within the range of $0.4 \%$ of the examined specimens. However, prevalence of Salmonella in this study is lower than the 7.3\% prevalence reported by Abu Elbashar (1996).

Apparently, intestinal contents are the highest Salmonella harboring parts demonstrating incidence of Salmonella spp. up to $11.4 \%$. This finding is in agreement with earlier reports that Salmonella concentrate in the gastrointestinal tract (Rasschaert et al., 2007; Van Schothorst and Notermans, 1980).

Eggs were second in rating of prevalence with a $7 \%$ incidence. Eggs are known as the main source of transmission of Salmonella to humans (Baumler et al., 2000). Liver and skin demonstrated similar rates of contamination with a value of 5\% prevalence. Ten $(5 \%)$ of skin specimens were found to be contaminated by Salmonella. Isolation of Salmonella spp. from poultry livers in this study is in agreement with reports of Procura et al. (2019).

The high rates of resistance reported in this study can be partially attributed to the widespread use of antibiotics agents given to poultry. The massive use of antimicrobial agents for medical and veterinary purposes creates a selective pressure which leads to an increasing development of antibiotic resistance worldwide (Mateu and Martin, 2001).

In this study resistance to Tetracycline was the most common resistance. Tetracycline has been one of the most commonly used antibiotics for animal production (Carraminana et al., 2004). Resistance to $\beta$-lactams antibiotics was manifested by one isolate showed resistance to amoxicillin-clavulanic-acid and ceftazidime.

Seventeen isolates $(44.7 \%)$ were found to be resistant to sulfonamides; compound sulfonamide and sulfamethoxazole-trimethoprim. The sulfonamides are broadspectrum antimicrobial agents, but their antimicrobial activity is significantly limited by the resistance that has developed after more than 50 years of use (Prescott, 2000).

\section{CONCLUSION}

This study showed that presence of Salmonella spp. in poultry and detection of eight serovars which are related to poultry. The high rates of resistance reported in this study can be partially attributed to the widespread use of antibiotics agents given to poultry. The massive use of antimicrobial agents for medical and veterinary purposes creates a selective pressure which leads to an increasing development of antibiotic resistance worldwide. The PFGE analysis showed different patterns.

\section{REFERRENCES}

Abu Elbashar, E. A. (1996). Isolation and Characterization of Salmonella from Domestic Fowl and its Environment in the State of Kordofan. M.Sc. Thesis, University of Khartoum, Sudan, 129 pp.

Baumler, A. J., Kusters, J. G., Stojiljkovic, I. \& Heffron, F. (1994). Salmonella Typhimurium loci involved in survival within macrophages. Infect. Immun, 62, 1623-1630.

Carraminana, J. J., Rota, C. A., Agustin, I. \& Herrera, A. (2004). High prevalence of multiple resistance to antibiotics in Salmonella serovars isolated from a poultry slaughterhouse in Spain. Vet. Microbiol, 104(1-2), 133-139. https://doi.org/10.1016/j.vetmic.2004.08.010

CDC (2002). Standardaized molecular subtyping of food borne bacterial pathogens by Pulsed -Field Gel Electrophoresis. Atlanta, GA., USA.

Fadlalla, I. M. T. (2003). Molecular Epidemiology of Multiple-Drug Resistant Salmonella from the Sudan. Ph.D. Thesis, University of Khartoum, Sudan, 198 pp.

Fung, F., Wang, H. S., \& Menon, S. (2018). Food safety in the $21^{\text {st }}$ century. Biomedical journal, 41(2), 88-95. https://doi.org/10.1016/j.bj.2018.03.003

Gal-Mor, O., Boyle, E. C., \& Grassl, G. A. (2014). Same species, different diseases: how and why typhoidal and non-typhoidal Salmonella enterica serovars differ. Front. Microbiol, 5, 391. https://dx.doi.org/10.3389\%2Ffmicb.2014.00391 Hur, J., Jawale, C., \& Lee, J. H. (2011). Antimicrobial resistance of Salmonella isolated from food animals: a review, 45, 819-830. Food Research International http://dx.doi.org/10.1016/j.foodres.2011.05.014.

Jajere, S. M. (2019). A review of Salmonella enterica with particular focus on the pathogenicity and virulence factors, host specificity and antimicrobial resistance including multidrug resistance. Vet World, 12, 504-521.
Malorny, B., Bunge, C., Hoorfar, J., \& Helmuth, R. (2003). Multicenter validation of the analytical accuracy of Salmonella PCR: towards an international standard. Appl. Environ. Microbiol, 69, 290-296. https://doi.org/10.1128/aem.69.1.290$\underline{296.2003}$

Mateu, E. \& Martin, M. (2001). Why is anti-microbial resistance a veterinary problem as well as? J. Vet. Med. B. Infect. Dis. Vet. Public Health, 48(8), 569581. https://doi.org/10.1046/j.1439-0450.2001.00475.x

NCCLS (2000). Performance standards for antimicrobial disc susceptibility tests, 7th edition, vol. 17, no.2. (Approved Standard M2-A7. NCCLS, Wayne, PA)

Popoff, M. Y. (2001). Antigenic Formulas of the Salmonella serovars. WHO Collaborating Centre for Reference and Research on Salmonella, $8^{\text {th }}$ Edition, Pasteur institute, Paris, France.

Prescott, J. F. (2000). Sulfonamides, diaminopyrimidines, and their combinations, in Prscott, j. F., Baggot, J. D. and walker, R. D. (2000). Antimicrobial Therapy in Veterinary Medicine. $3^{\text {rd }}$.Edition. Ames, IA., Iowa State University Press. pp. 290314

Procura, F., Bueno, D. J., Bruno, S. B. \& Rogé, A. D. (2019). Prevalence, antimicrobial resistance profile and comparison of methods for the isolation of Salmonella in chicken liver from Argentina. Food Res. Int, 119, 541-546. https://doi.org/10.1016/j.foodres.2017.08.008

Rasschaert, G., Houf, K., Van Hende, J.m \& De Zutter, L. (2007). Investigation of the concurrent colonization with Campylobacter and Salmonella in poultry flocks and assessment of sampling site for status determination at slaughter. Vet. Microbiol, 123(1-3), 104-109. https://doi.org/10.1016/j.vetmic.2007.03.011

Shi, C., Singh, P., Ranieri, M. L, Wiedmann, M., Moreno., \& Switt A. I. (2015). Molecular methods for serovar determination of Salmonella. Critical Reviews Microbiology, 41(3), 309-325. https://doi.org/10.3109/1040841X.2013.837862

Su, L. H., \& Chiu, C. H. (2007). Salmonella: Clinical importance and evolution of nomenclature. Chang Gung Med J, 30(3), 210-219.

Tadee, P., Boonkhot, P., Pornruangwong, S., \& Patchanee, P. (2015). Comparative phenotypic and genotypic characterization of Salmonella spp. in pig farms and slaughterhouses in two provinces in northern Thailand. PloS one, 18, 10(2), e0116581 https://doi.org/10.1371/journal.pone.0116581

Van Schothorst, M. \& Notermans, S. (1980). Food-borne diseases associated with poultry, in Mead, G. C. and Freeman, B. M. eds., Meat Quality in Poultry and Game Birds, British poultry science Ltd, Edinburgh, pp. 79-90.

Yagoub, I. A. (1986). Isolation and Characterization of Salmonella from the Domestic Fowl and its Environment. M. V. Sc. Thesis, University of Khartoum, Sudan. 110 pp.

Zishiri, O., Mkhize, N., \& Mukaratirwa, S. (2016). Prevalence of virulence and antimicrobial resistance genes in Salmonella spp. isolated from commercial chickens and human clinical isolates from South Africa and Brazil. Onderstepoort. $J$ Vet Res, 83, 1-11. https://doi.org/10.4102/ojvr.v83i1.1067 